\title{
Effects of Reneging, Server Breakdowns and Vacation on a Batch Arrival Single Server Queueing System with Three Fluctuating Modes of Service
}

\author{
Samuel Ugochukwu Enogwe1 ${ }^{*}$, Happiness Onyebuchi Obiora-Ilouno ${ }^{2}$ \\ ${ }^{1}$ Department of Statistics, Michael Okpara University of Agriculture, Umudike, Nigeria \\ ${ }^{2}$ Department of Statistics, Nnamdi Azikiwe University, Awka, Nigeria \\ Email: ^senogwe@yahoo.com, obiorailounoho@yahoo.com
}

How to cite this paper: Enogwe, S.U. and Obiora-Ilouno, H.O. (2020) Effects of Reneging, Server Breakdowns and Vacation on a Batch Arrival Single Server Queueing System with Three Fluctuating Modes of Service. Open Journal of Optimization, 9 , 105-128.

https://doi.org/10.4236/ojop.2020.94008

Received: September 13, 2020

Accepted: December 6, 2020

Published: December 9, 2020

Copyright $\odot 2020$ by author(s) and Scientific Research Publishing Inc. This work is licensed under the Creative Commons Attribution International License (CC BY 4.0).

http://creativecommons.org/licenses/by/4.0/

(c) (i) Open Access

\begin{abstract}
This article examines the effects of reneging, server breakdown and server vacation on the various states of the batch arrivals queueing system with single server providing service to customers in three fluctuating modes. In this queueing system, any batch arrival joins the queue if the server is busy or on vacation or under repair. However, if the server is free, one customer from the arriving batch joins the service immediately while others join the queue. In case of server breakdown, the customer whose service is interrupted returns back to the head of the queue. As soon as the server has is repaired, the server attends to the customer in mode 1 . For this queueing system, customers that are impatient due to breakdown and server vacation may renege (leave the queue without getting service). Due to fluctuating modes of service delivery, the system may provide service with complete or reduced efficiency. Consequently, we construct the mathematical model and derive the probability generating functions of the steady state probabilities of several states of the system including the steady state queue size distribution. Further, we discuss some particular cases of the proposed queueing model. We present numerical examples in order to demonstrate the effects of server vacation and reneging on the various states of the system. The study revealed that an increase in reneging and a decrease in server vacation results in a decrease in server utilization and an increase in server's idle time provided rates of server breakdown and repair completion are constant. In addition, the probability of server vacation, the probability of system is under repair and the probabilities that the server provides service in three fluctuating modes decreases due to an increase in reneging and a decrease in vacation completion rates.
\end{abstract}




\section{Keywords}

Queueing, Reneging, Server Vacation, Server Breakdowns, Fluctuating Modes of Service

\section{Introduction}

A batch arrival single server queueing system is one in which customers arrive for service in groups or in batches and are served individually by one server. Some examples of batch arrivals include families that go to a restaurant for lunch in a particular period of the day, convoy of a political officer that came to a filling station for refuelling and sets of triplets brought to a hospital for medical treatment. Other examples are batch of raw materials supplied to industry for manufacturing, a group of imported items to be unloaded at a warehouse and so on.

Many batch arrival single server queuing systems assume that the server offers one type of general service at the same average service rate. However, in practice, average rate of service may vary due to several reasons. For example, the speed of internet on a particular Android phone may not be the same due to network fluctuations. In petrol stations, the fuel pumps do not always refuel tanks at the same rate due to fluctuations in electric power or due to pump's efficiency. In addition, climatic conditions as well as other unforeseen circumstances may affect the performance of radio networks and as such, communication companies cannot provide service to their customers at the same average rate.

In view of the aforementioned reasons for variation in average service rate, the mode of service delivery becomes fluctuating. Obviously, the fluctuating mode of service delivery affects the efficiency of a queueing system and one is required to develop queueing models for fluctuating modes of service. To this end, Baruah et al. [1] introduced a batch arrival single server queuing system in which the server provides general service in two fluctuating modes. Similarly, Madan [2] proposed and studied a batch arrival single server queueing system for providing general service in three fluctuating modes. [3] [4] studied a bulk arrivals retrial queue with fluctuating modes of service. Similarly, [5] analyzed a queueing model with fluctuating modes of service. Further, [6] introduced a non-markovian single-server retrial queueing system with fluctuating modes of service. Additionally, [7] analyzed a batch arrival queueing system with single server providing service to a batch of customers with dissimilar rates in two fluctuating modes of service.

Apart from the fluctuating modes of service delivery, a queueing system may experience a sudden breakdown, which causes the stoppage of service until the machine is fixed. In such a situation, the customer whose service is interrupted returns back to the head of the queue and waits until repair process is completed. In reality, random breakdowns usually occur in machines used for production 
and manufacturing units, communication systems, traffic intersections, automated teller machines and so on. Batch arrival queues with breakdowns have been extensively studied by [8] [9] [10] [11] [12] among others.

Obviously, during the period of system breakdown, the server may proceed on vacation. By vacation, we mean that the server becomes unavailable for a random period of time from its primary customers to serve elsewhere [13]. Works by [14]-[21] deals extensively on vacation queueing systems.

One of the consequences of the fluctuating modes of service, server breakdown and server vacation is that they generally slow down the service time of customers and increase their waiting time. Consequently, customers who have been waiting in the queue for a long time than anticipated often become impatient and leave the queue without getting service. This kind of customer behaviour refers to reneging. Ideally, reneging is a practical experience in most queueing systems because not all customers that enter the queue wait to receive service. Queue with reneging has been discussed in [22]-[29] among others.

From the foregoing, it is quite evident that there is no model in the queueing literature that combines batch arrivals, three fluctuating modes of service, server breakdown, server vacation and reneging. This kind of model has potential applications in a production line, where raw materials arrive in batches of random size instead of as single units. The machine producing an item may require three fluctuating modes of service such as processing of raw material in fast, normal or slow modes. Also, the machine producing items may suddenly breakdown due to mechanical or job related problems and as such the process is stopped either for preliminary checks of raw materials or for the maintenance or repair. During the period of server breakdown and server vacation, customers that are not satisfied with the time wastage leave the queue. Thus, the aim of this paper is to develop a model that allows the server to exhibit all these features highlighted to make the queueing model more realistic and flexible in studying the real world queueing situations.

\section{Mathematical Description of the Model}

The following assumptions describe the proposed queueing system:

1) Customers arrive at the system in batches of variable size in accordance with a compound Poisson process. Let the arrival batch size $Y$ be a random variable with probability mass function $P(Y=i)=c_{i}, i=1,2,3, \cdots$ Then $\lambda c_{i} \mathrm{~d} t(i=1,2,3, \cdots)$ denotes the first order probability that a batch of $i$ customers arrives at the system during a short interval of time $(t, t+\mathrm{d} t]$, where $0 \leq c_{i} \leq 1, \sum_{i=1}^{\infty} c_{i}=1$ and $\lambda>0$ is the average arrival rate of batches.

2) There is one server providing service in three fluctuating modes. Customers receive service one by one based on first-come, first-served (FCFS) queue discipline. Further, the probability of the server providing service in mode 1 , mode 2 and mode 3 are $p_{1}, p_{2}$ and $p_{3}$ respectively, where $\left(p_{1}+p_{2}+p_{3}=1\right)$. The 
service times at the three different fluctuation modes of service follow different general (arbitrary) distributions with distribution functions $G_{j}(s)$ and the density functions $g_{j}(s), j=1,2,3$. Let $\mu_{j}(y)$ dy be the conditional probability density of completion of service mode $j(j=1,2,3)$ of services during the interval $(y, y+\mathrm{d} y]$, given that elapsed time is $y$, so that

$$
\mu_{j}(y)=\frac{g_{j}(y)}{1-G_{j}(y)}, j=1,2,3
$$

and therefore

$$
g_{j}(s)=\mu_{j}(s) \mathrm{e}^{-\int \mu_{j} \mu_{j}(y) \mathrm{d} y}, j=1,2,3
$$

3) The system may fail or be subjected to breakdown at random. The breakdowns are time-homogeneous in the sense that the server can fail even while it is idle. The customer receiving service during breakdown comes back to the head of the queue. In this study, it is assumed that time between breakdowns occur according to Poisson probability law with average rate of breakdown $\alpha>0$. Consequently, $\alpha \mathrm{d} t$ denotes the first order probability that the system will breakdown during the interval $(t, t+\mathrm{d} t]$. Once the system breaks down, it enters a repair process immediately. As soon as the repair process is completed, the server immediately provides service to the customer in mode 1 with probability $p_{1}$. The repair times follow a general (arbitrary) distribution with distribution function $R(h)$ and density function $r(h)$. Let $\beta(y)$ dy be the conditional probability of completion of the repair process during the interval $(y, y+\mathrm{d} y]$ be such that

$$
\beta(y)=\frac{r(y)}{1-R(y)}
$$

and therefore,

$$
r(h)=\beta(h) \mathrm{e}^{-\int^{-h} \beta(y) \mathrm{d} y}
$$

4) After each service completion, the server may take a vacation of a random length with probability $\phi$ or may continue to serve the next customer with probability $(1-\phi)$. On returning from vacation, the server instantly starts serving the customer at the head of the queue, if any. The server's vacation times follow general (arbitrary) distribution, with distribution function $V(\omega)$ and probability density function $w(v)$. Let $\phi(y)$ dy be the conditional probability of completion of a vacation period during the interval $(y, y+d y]$, given that the elapsed vacation time is $y$, so that

$$
\phi(y)=\frac{v(y)}{1-V(y)}
$$

and therefore,

$$
v(\omega)=\phi(\omega) \mathrm{e}^{-\iint^{-} \phi(y) \mathrm{d} y}
$$


5) Due to server vacations, customers waiting in line may become impatient and renege (leave the queue). Reneging is therefore assumed to follow the exponential distribution with parameter $\gamma>0$. Thus, $f(t)=\gamma \mathrm{e}^{-\gamma t}, \gamma>0$. Consequently, $\gamma \mathrm{d} t$ represents the probability that an arriving batch of customers' reneges during a short during the interval $(t, t+\mathrm{d} t]$.

6) Various stochastic processes involved in the system are assumed to be independent of each other.

\section{Definitions, Notations and Equations Governing the Proposed Queueing Model}

\subsection{Definitions and Notations}

Let $N_{q}(t)$ denote the queue size (excluding one in service) at time $t, G_{j}^{0}(t)$ be the elapsed service time of the customer in jh mode of service at time $t$, where $j=1,2,3$. In addition, let $V^{0}(t)$ and $R^{0}(t)$ be the elapsed vacation time and repair time of the server respectively. Thus, we introduce the variable $Y(t)$ as follows:

$$
Y(t)=\left\{\begin{array}{l}
0 \text { if the server is idle at time } t \\
1 \text { if the server is providing service in mode } 1 \text { at time } t \\
2 \text { if the server is providing service in mode } 2 \text { at time } t \\
3 \text { if the server is providing service in mode } 3 \text { at time } t \\
4 \text { if the server is on vacation at time } t \\
5 \text { if the server is under repair during breakdown at time } t
\end{array}\right.
$$

Thus, the supplementary variable $G_{1}^{0}(t), G_{2}^{0}(t), G_{3}^{0}(t), V^{0}(t)$ and $R^{0}(t)$ are introduced in order to obtain a bivariate Markov process $\left\{N_{q}(t), X(t)\right\}$, where $X(t)=0$ if $Y(t)=0, X(t)=G_{1}^{0}(t)$ if $Y(t)=1$, $X(t)=G_{2}^{0}(t)$ if $Y(t)=2, \quad X(t)=G_{3}^{0}(t)$ if $Y(t)=3, \quad X(t)=V^{0}(t)$ if $Y(t)=4, X(t)=R^{0}(t)$ if $Y(t)=5$. Next, the limiting probabilities are given by:

$$
\begin{aligned}
& P_{n}^{(1)}(y)=\lim _{t \rightarrow \infty} P\left[N_{q}(t)=n, X(t)=G_{1}^{0}(t) ; y<G_{1}^{0}(t) \leq y+\mathrm{d} y\right], y>0, n \geq 0 \\
& P_{n}^{(2)}(y)=\lim _{t \rightarrow \infty} P\left[N_{q}(t)=n, X(t)=G_{2}^{0}(t) ; y<G_{2}^{0}(t) \leq y+\mathrm{d} y\right], y>0, n \geq 0 \\
& P_{n}^{(3)}(y)=\lim _{t \rightarrow \infty} P\left[N_{q}(t)=n, X(t)=G_{3}^{0}(t) ; y<G_{3}^{0}(t) \leq y+\mathrm{d} y\right], y>0, n \geq 0 \\
& V_{n}(y)=\lim _{t \rightarrow \infty} P\left[N_{q}(t)=n, X(t)=V^{0}(t) ; y<V^{0}(t) \leq y+\mathrm{d} y\right], y>0, n \geq 0 \\
& R_{n}(y)=\lim _{t \rightarrow \infty} P\left[N_{q}(t)=n, X(t)=R^{0}(t) ; y<R^{0}(t) \leq y+\mathrm{d} y\right], y>0, n \geq 0
\end{aligned}
$$

Further, it may be noted that since it is assumed $V(y), R(y)$ and $G_{j}(y)$ are distribution functions, then $R(0)=0, R(\infty)=1, V(0)=0, V(\infty)=1$, $G_{j}(0)=0, G_{j}(\infty)=1$ for $j=1,2,3$, and that $V(y), R(y)$ and $G_{j}(y)$ are continuous at $y=0$, so that

$$
\beta(y) \mathrm{d} y=\frac{\mathrm{d} R(y)}{1-R(y)}, \phi(y) \mathrm{d} y=\frac{\mathrm{d} V(y)}{1-V(y)} \text { and } \mu_{j}(y) \mathrm{d} y=\frac{\mathrm{d} G_{j}(y)}{1-G_{j}(y)}
$$


are the first order differential(hazard rate) functions of $R, V$ and $G_{j}(j=1,2,3)$, respectively.

Assume that the system is in steady state condition (i.e., the normal condition that a queueing system is in after operating for some time with a fixed utilization factor less than one). Define $P_{n}^{(j)}(y)$ as the steady state probability that the server is active providing service in mode $j(j=1,2,3)$ and there are $n(n \geq 0)$ customers in the queue excluding the one customer in service and the elapsed service time of this customer is $y$. Accordingly, $P_{n, j}=\int_{0}^{\infty} P_{n, j}(y)$ dy denotes the corresponding steady state probability that there are $n(n \geq 1)$ customers in the queue excluding the one receiving service in mode $j(j=1,2,3)$ irrespective of the elapsed service time $y$ of this customer; $R_{n}(y)$ denotes steady state probability that the server is under repairs since the elapsed repair time $y$ and there are $n(n \geq 0)$ customers in the queue. Accordingly, $R_{n}=\int_{0}^{\infty} R_{n}(y) \mathrm{d} y$ denotes the corresponding steady state probability that the server is under repairs and there are $n(n \geq 0)$ customers in the queue irrespective of the elapsed repair time $y$ of the server; $V_{n}(y)$ denotes steady state probability that the server is on vacation with elapsed vacation time $y$ and there are $n(n \geq 0)$ customers in the queue. Accordingly, $V_{n}=\int_{0}^{\infty} V_{n}(y) \mathrm{d} y$ denotes the corresponding steady state probability that there are $n(n \geq 0)$ customers in the queue and the server is on vacation irrespective of the elapsed vacation time $y$ of the server; and $Q$ denotes steady state probability that there are no customers in the system and the server is idle but available in the system.

Next, the probability generating functions (PGFs) used in this paper is as given:

$$
\left.\begin{array}{l}
P^{(j)}(x, z)=\sum_{n=1}^{\infty} z^{n} P_{n}^{(j)}(y) ; P_{j}(z)=\sum_{n=1}^{\infty} z^{n} P_{n}^{(j)}, j=1,2,3 \\
R(x, z)=\sum_{n=1}^{\infty} z^{n} R_{n}(y) ; R(z)=\sum_{n=1}^{\infty} z^{n} R_{n} \\
V(x, z)=\sum_{n=1}^{\infty} z^{n} V_{n}(y) ; V(z)=\sum_{n=1}^{\infty} z^{n} V_{n} \\
C(z)=\sum_{i=1}^{\infty} z^{i} c_{i}
\end{array}\right\}
$$

which are convergent inside the circle given by $|z| \leq 1$. Also the Laplace-Stieltjes transform of a function $F(t)$ is defined as:

$$
F^{*}(s)=\int_{0}^{\infty} \mathrm{e}^{-s t} \mathrm{~d} F(t)
$$

\subsection{Governing Equations of the Proposed Queueing Model}

The steady state equations governing the proposed queueing model are as follows: 


$$
\begin{gathered}
\frac{\mathrm{d}}{\mathrm{d} y} P_{n}^{(1)}(y)+\left(\lambda+\mu_{1}(y)+\alpha\right) P_{n}^{(1)}(y)=\lambda \sum_{i=1}^{n} c_{i} P_{n-i}^{(1)}(y), n \geq 1 \\
\frac{\mathrm{d}}{\mathrm{d} y} P_{0}^{(1)}(y)+\left(\lambda+\mu_{1}(y)+\alpha\right) P_{0}^{(1)}(y)=0 \\
\frac{\mathrm{d}}{\mathrm{d} y} P_{n}^{(2)}(y)+\left(\lambda+\mu_{2}(y)+\alpha\right) P_{n}^{(2)}(y)=\lambda \sum_{i=1}^{n} c_{i} P_{n-i}^{(2)}(y), n \geq 1 \\
\frac{\mathrm{d}}{\mathrm{d} y} P_{0}^{(2)}(y)+\left(\lambda+\mu_{2}(y)+\alpha\right) P_{0}^{(2)}(y)=0 \\
\frac{\mathrm{d}}{\mathrm{d} y} P_{n}^{(3)}(y)+\left(\lambda+\mu_{3}(y)+\alpha\right) P_{n}^{(3)}(y)=\lambda \sum_{i=1}^{n} c_{i} P_{n-i}^{(3)}(y), n \geq 1 \\
\frac{\mathrm{d}}{\mathrm{d} y} R_{n}(y)+(\lambda+\beta(y)+\gamma) R_{n}(y)=\lambda \sum_{i=1}^{n} c_{i} R_{n-i}(y)+\gamma R_{n+1}(y), n \geq 1 \\
\frac{\mathrm{d}}{\mathrm{d} y} R_{0}(y)+(\lambda+\beta(y)+\gamma) R_{0}(y)=\gamma R_{1}(y) \\
+\int_{0}^{\infty} R_{0}(y) \beta(y) \mathrm{d} y+\int_{0}^{\infty} V_{0}(y) \phi(y) \mathrm{d} y \\
\frac{\mathrm{d}}{\mathrm{d} y} V_{n}(y)+(\lambda+\phi(y)+\gamma) V_{n}(y)=\lambda \sum_{i=1}^{n} c_{i} V_{n-i}(y)+\gamma V_{n+1}(y), n \geq 1 \\
\frac{\mathrm{d}}{\mathrm{d} y} V_{0}(y)+(\lambda+\phi(y)+\gamma) V_{0}(y)=\gamma V_{1}(y) \\
\left.=(1-p) \int_{0}^{\infty} P_{0}^{(1)}(y) \mu_{1}(y) \mathrm{d} y+\int_{0}^{\infty} P_{0}^{(2)}(y) \mu_{2}(y) \mathrm{d} y+\int_{0}^{\infty} P_{0}^{(3)}(y) \mu_{3}(y) \mathrm{d} y\right]
\end{gathered}
$$

The governing Equations (9)-(19) are to be solved subject to the boundary conditions given below at $y=0$ :

$$
\begin{aligned}
P_{n}^{(1)}(0)= & \lambda c_{n+1} Q+(1-p)\left[\pi_{1} \int_{0}^{\infty} P_{n+1}^{(1)}(y) \mu_{1}(y) \mathrm{d} y\right. \\
& \left.+\pi_{1} \int_{0}^{\infty} P_{n+1}^{(2)}(y) \mu_{2}(y) \mathrm{d} y+\pi_{1} \int_{0}^{\infty} P_{n+1}^{(3)}(y) \mu_{3}(y) \mathrm{d} y\right] \\
& +\pi_{1} \int_{0}^{\infty} R_{n+1} \beta(y) \mathrm{d} y+\pi_{1} \int_{0}^{\infty} V_{n+1} \phi(y) \mathrm{d} y, n \geq 0 \\
P_{n}^{(2)}(0)= & \lambda c_{n+1} \pi_{2} Q+(1-p)\left[\pi_{2} \int_{0}^{\infty} P_{n+1}^{(1)}(y) \mu_{1}(y) \mathrm{d} y\right. \\
& \left.+\pi_{2} \int_{0}^{\infty} P_{n+1}^{(2)}(y) \mu_{2}(y) \mathrm{d} y+\pi_{2} \int_{0}^{\infty} P_{n+1}^{(3)}(y) \mu_{3}(y) \mathrm{d} y\right] \\
& +\pi_{2} \int_{0}^{\infty} R_{n+1} \beta(y) \mathrm{d} y+\pi_{2} \int_{0}^{\infty} V_{n+1} \phi(y) \mathrm{d} y, n \geq 0
\end{aligned}
$$




$$
\begin{gathered}
P_{n}^{(3)}(0)=\lambda c_{n+1} \pi_{3} Q+(1-p)\left[\pi_{3} \int_{0}^{\infty} P_{n+1}^{(1)}(y) \mu_{1}(y) \mathrm{d} y\right. \\
\left.+\pi_{3} \int_{0}^{\infty} P_{n+1}^{(2)}(y) \mu_{2}(y) \mathrm{d} y+\pi_{3} \int_{0}^{\infty} P_{n+1}^{(3)}(y) \mu_{3}(y) \mathrm{d} y\right] \\
+\pi_{3} \int_{0}^{\infty} R_{n+1} \beta(y) \mathrm{d} y+\pi_{3} \int_{0}^{\infty} V_{n+1} \phi(y) \mathrm{d} y, n \geq 0 \\
R_{n+1}(0)=\alpha \int_{0}^{\infty} P_{n}^{(1)}(y) \mathrm{d} y+\alpha \int_{0}^{\infty} P_{n}^{(2)}(y) \mathrm{d} y+\alpha \int_{0}^{\infty} P_{n}^{(3)}(y) \mathrm{d} y, n \geq 0 \\
V_{n}(0)=p\left[\int_{0}^{\infty}(0)=\alpha Q\right. \\
\left.\int_{0}^{(1)}(y) \mu_{1}(y) \mathrm{d} y+\int_{0}^{\infty} P_{n}^{(2)}(y) \mu_{2}(y) \mathrm{d} y+\int_{0}^{\infty} P_{n}^{(3)}(y) \mu_{3}(y) \mathrm{d} y\right], n \geq 0
\end{gathered}
$$

\section{Main Results}

\subsection{Queue Size Distribution at Random Epoch for the Proposed Queueing Model}

Theorem 1. Under the stability condition $\rho<1$, the proposed queueing model has the marginal probability generating functions for the server's state queue size defined by Equations (70)-(74).

Proof. Multiplying Equation (9) by $z^{n}$, summing both sides over $n$ from 1 to $\infty$, adding the results to Equation (10) and utilizing the probability generating functions defined in Equation (7) gives

$$
\frac{\mathrm{d}}{\mathrm{d} y} P^{(1)}(y, z)+\left[\lambda-\lambda C(z)+\mu_{1}(y)+\alpha\right] P^{(1)}(y, z)=0
$$

Similarly, multiply Equation (11) by $z^{n}$, take the sum of both sides over $n$ from 1 to $\infty$, add the result to Equation (12) and use the probability generating functions defined in Equation (7) to obtain

$$
\frac{\mathrm{d}}{\mathrm{d} y} P^{(2)}(y, z)+\left[\lambda-\lambda C(z)+\mu_{2}(y)+\alpha\right] P^{(2)}(y, z)=0
$$

Multiplying Equation (13) by $z^{n}$, taking sum of both sides over $n$ from 1 to $\infty$, adding the result to Equation (14) and making use of the probability generating functions defined in Equation (7) yields

$$
\frac{\mathrm{d}}{\mathrm{d} y} P^{(3)}(y, z)+\left[\lambda-\lambda C(z)+\mu_{3}(y)+\alpha\right] P^{(3)}(y, z)=0
$$

Multiplying Equation (15) by $z^{n}$, taking sum of both sides over $n$ from 1 to $\infty$, adding the result to Equation (16) and using the probability generating functions defined in Equation (7), one obtains

$$
\frac{\mathrm{d}}{\mathrm{d} y} R(y, z)+\left(\lambda-\lambda C(\mathrm{z})+\beta(y)+\gamma-\frac{\gamma}{\mathrm{z}}\right) R(y, z)=0
$$

Multiplying Equation (17) by $z^{n}$, taking sum of both sides over $n$ from 1 to $\infty$, adding the result to Equation (18) and utilizing the probability generating functions defined in Equation (7) leads to 


$$
\frac{\mathrm{d}}{\mathrm{d} y} V(y, z)+\left(\lambda-\lambda C(z)+\eta(y)+\gamma-\frac{\gamma}{z}\right) V(y, z)=0
$$

Integrating Equations (26)-(30) with respect to $y$ between the limits 0 to $y$ gives

$$
\begin{aligned}
P^{(1)}(y, z) & =P^{(1)}(0, z) \mathrm{e}^{-(\lambda-\lambda C(z)+\alpha) y-\int_{0}^{y} \mu_{1}(t) d t} \\
P^{(2)}(y, z) & =P^{(2)}(0, z) \mathrm{e}^{-(\lambda-\lambda C(z)+\alpha) y-\int_{0}^{y} \mu_{2}(t) d t} \\
P^{(3)}(y, z) & =P^{(3)}(0, z) \mathrm{e}^{-(\lambda-\lambda C(z)+\alpha) y-\int_{0}^{y} \mu_{3}(t) d t} \\
R(y, z) & =R(0, z) \mathrm{e}^{-\left(\lambda-\lambda C(z)+\gamma-\frac{\gamma}{z}\right)-\int_{0}^{y} \beta(t) d t} \\
V(y, z) & =V(0, z) \mathrm{e}^{-\left(\lambda-\lambda C(z)+\gamma-\frac{\gamma}{z}\right)-\int_{0}^{y} \eta(t) d t}
\end{aligned}
$$

Now, for the boundary conditions, multiply Equation (20) by $z^{n+1}$, take the sum of both sides over $n$ from 0 to $\infty$ and use Equation (19) as well as the probability generating functions defined in Equation (7) to obtain

$$
\begin{aligned}
z P^{(1)}(0, z)= & p_{1}(\lambda C(z)-\lambda-\alpha) Q+(1-\phi) p_{1}\left[\int_{0}^{\infty} P^{(1)}(y, z) \mu_{1}(y) d y\right. \\
& \left.+\int_{0}^{\infty} P^{(2)}(y, z) \mu_{2}(y) \mathrm{d} y+\int_{0}^{\infty} P^{(3)}(y, z) \mu_{3}(y) \mathrm{d} y\right] \\
& +p_{1} \int_{0}^{\infty} R(y, z) \beta(y) \mathrm{d} y+p_{1} \int_{0}^{\infty} V(y, z) \phi(y) \mathrm{d} y
\end{aligned}
$$

Similarly, multiply Equation (21) by $z^{n+1}$, take the sum over of both sides $n$ from 0 to $\infty$ and use Equation (19) as well as the probability generating functions defined in Equation (7), we have

$$
\begin{aligned}
z P^{(2)}(0, z)= & p_{2}(\lambda C(z)-\lambda-\alpha) Q+(1-\phi) p_{2}\left[\int_{0}^{\infty} P^{(1)}(y, z) \mu_{1}(y) d y\right. \\
& \left.+\int_{0}^{\infty} P^{(2)}(y, z) \mu_{2}(y) \mathrm{d} y+\int_{0}^{\infty} P^{(3)}(y, z) \mu_{3}(y) \mathrm{d} y\right] \\
& +p_{2} \int_{0}^{\infty} R(y, z) \beta(y) \mathrm{d} y+p_{2} \int_{0}^{\infty} V(y, z) \phi(y) \mathrm{d} y
\end{aligned}
$$

Multiplying Equation (22) by $z^{n+1}$, taking the sum over $n$ from 0 to $\infty$ and using Equation (19) as well as the probability generating functions defined in Equation (7) one gets

$$
\begin{aligned}
z P^{(3)}(0, z)= & p_{3}(\lambda C(z)-\lambda-\alpha) Q+(1-\phi) p_{3}\left[\int_{0}^{\infty} P^{(1)}(y, z) \mu_{1}(y) \mathrm{d} y\right. \\
& \left.+\int_{0}^{\infty} P^{(2)}(y, z) \mu_{2}(y) \mathrm{d} y+\int_{0}^{\infty} P^{(3)}(y, z) \mu_{3}(y) \mathrm{d} y\right] \\
& +p_{3} \int_{0}^{\infty} R(y, z) \beta(y) \mathrm{d} y+p_{3} \int_{0}^{\infty} V(y, z) \phi(y) \mathrm{d} y
\end{aligned}
$$


Multiplying Equation (23) by $z^{n+1}$, taking the sum of both sides over $n$ from 0 to $\infty$ and using Equation (24) as well as the probability generating functions defined in Equation (7) leads to

$$
\begin{aligned}
R(0, z)-\alpha Q= & \alpha z \int_{0}^{\infty} P^{(1)}(y, z) \mu_{1}(y) \mathrm{d} y+\alpha z \int_{0}^{\infty} P^{(2)}(y, z) \mu_{2}(y) \mathrm{d} y \\
& +\alpha z \int_{0}^{\infty} P^{(3)}(y, z) \mu_{3}(y) \mathrm{d} y \\
R(0, z) & =\alpha z\left[P^{(1)}(z)+P^{(2)}(z)+P^{(3)}(z)\right]+\alpha Q
\end{aligned}
$$

Again, multiplying Equation (25) by $z^{n+1}$, taking the sum over $n$ from 0 to $\infty$ and using the probability generating functions defined in Equation (7) yields:

$V(0, z)=\phi\left[\int_{0}^{\infty} P^{(1)}(y, z) \mu_{1}(y) \mathrm{d} y+\int_{0}^{\infty} P^{(2)}(y, z) \mu_{2}(y) \mathrm{d} y+\int_{0}^{\infty} P^{(3)}(y, z) \mu_{3}(y) \mathrm{d} y\right]$

Integrating Equations (31)-(35) by parts with respect to $y$ between the limits 0 and $\infty$ yields

$$
\begin{aligned}
& P^{(1)}(z)=P^{(1)}(0, z) \frac{\left[1-S_{1}^{*}(\lambda-\lambda C(z)+\alpha)\right]}{(\lambda-\lambda C(z)+\alpha)} \\
& P^{(2)}(z)=P^{(2)}(0, z) \frac{\left[1-S_{2}^{*}(\lambda-\lambda C(z)+\alpha)\right]}{(\lambda-\lambda C(z)+\alpha)} \\
& P^{(3)}(z)=P^{(3)}(0, z) \frac{\left[1-S_{3}^{*}(\lambda-\lambda C(z)+\alpha)\right]}{(\lambda-\lambda C(z)+\alpha)} \\
& R(z)=R(0, z) \frac{\left[1-B^{*}(\lambda-\lambda C(z)+\gamma-\gamma / z)\right]}{(\lambda-\lambda C(z)+\gamma-\gamma / z)} \\
& V(z)=V(0, z) \frac{1-F^{*}(\lambda-\lambda C(z)+\gamma-\gamma / z)}{(\lambda-\lambda C(z)+\gamma-\gamma / z)}
\end{aligned}
$$

where

$$
\begin{gathered}
G_{1}^{*}(\lambda-\lambda C(z)+\alpha)=\int_{0}^{\infty} \mathrm{e}^{-(\lambda-\lambda C(z)+\alpha) y} \mathrm{~d} G_{1}(y) \\
G_{2}^{*}(\lambda-\lambda C(z)+\alpha)=\int_{0}^{\infty} \mathrm{e}^{-(\lambda-\lambda C(z)+\alpha) y} \mathrm{~d} G_{2}(y) \\
G_{3}^{*}(\lambda-\lambda C(z)+\alpha)=\int_{0}^{\infty} \mathrm{e}^{-(\lambda-\lambda C(z)+\alpha) y} \mathrm{~d} G_{3}(y) \\
B^{*}(\lambda-\lambda C(z)+\gamma-\gamma / z)=\int_{0}^{\infty} \mathrm{e}^{-(\lambda-\lambda C(z)+\gamma-\gamma / z) y} \mathrm{~d} B(y) \\
F^{*}(\lambda-\lambda C(z)+\gamma-\gamma / z)=\int_{0}^{\infty} \mathrm{e}^{-(\lambda-\lambda C(z)+\gamma-\gamma / z) y} \mathrm{~d} F(y)
\end{gathered}
$$

are the Laplace-Steiltjes transform of the service time in the first, second, third 
modes of service, vacation time and repair time respectively.

To further simply Equations (36)-(40), multiply Equation (31) by $\mu_{1}(y)$; Equation (32) by $\mu_{2}(y)$; Equation (33) by $\mu_{3}(y)$, Equation (34) by $\beta(y)$ and Equation (35) by $\phi(y)$ respectively and integrate the results with respect to $y$ between the limits 0 and $\infty$, one gets

$$
\begin{aligned}
& \int_{0}^{\infty} P^{(1)}(y, z) \mu_{1}(y) \mathrm{d} y=P^{(1)}(0, z) S_{1}^{*}(\lambda-\lambda C(z)+\alpha) \\
& \int_{0}^{\infty} P^{(2)}(y, z) \mu_{2}(y) \mathrm{d} y=P^{(2)}(0, z) S_{2}^{*}(\lambda-\lambda C(z)+\alpha) \\
& \int_{0}^{\infty} P^{(3)}(y, z) \mu_{3}(y) \mathrm{d} y=P^{(3)}(0, z) S_{3}^{*}(\lambda-\lambda C(z)+\alpha) \\
& \int_{0}^{\infty} R(y, z) R(y) \mathrm{d} y=R(0, z) B^{*}\left(\lambda-\lambda C(z)+\gamma-\frac{\gamma}{z}\right) \\
& \int_{0}^{\infty} V(y, z) \phi(y) \mathrm{d} y=V(0, z) F^{*}\left(\lambda-\lambda C(z)+\gamma-\frac{\gamma}{z}\right)
\end{aligned}
$$

Let $\lambda-\lambda C(z)+\alpha=m, \quad \lambda-\lambda C(z)+\gamma-\gamma / z=k$ and substitute Equations (51)-(55) into Equation (36), (37) and (38) results to

$$
\begin{aligned}
& {\left[z-(1-\phi) p_{1} S_{1}^{*}(m)\right] P^{(1)}(0, z)-(1-\phi) p_{1} P^{(2)}(0, z) S_{2}^{*}(m)} \\
& -(1-\phi) p_{1} P^{(3)}(0, z) S_{3}^{*}(m)-p_{1} R(0, z) B^{*}(k)-p_{1} V(0, z) F^{*}(k)=-p_{1} m Q \\
& {\left[z-(1-\phi) p_{2} S_{2}^{*}(m)\right] P^{(2)}(0, z)-(1-\phi) p_{2} P^{(1)}(0, z) S_{1}^{*}(m)} \\
& -(1-\phi) p_{2} P^{(3)}(0, z) S_{3}^{*}(m)-p_{2} R(0, z) B^{*}(k)-p_{2} V(0, z) F^{*}(k)=-p_{2} m Q \\
& {\left[z-(1-\phi) p_{3} S_{3}^{*}(m)\right] P^{(3)}(0, z)-(1-\phi) p_{3} P^{(1)}(0, z) S_{1}^{*}(m)} \\
& -(1-\phi) p_{3} P^{(3)}(0, z) S_{3}^{*}(m)-p_{3} R(0, z) B^{*}(k)-p_{3} V(0, z) F^{*}(k)=-p_{3} m Q
\end{aligned}
$$

Inserting Equations (41), (42) and (43) into Equation (39), we get

$$
\begin{aligned}
& \alpha z\left[\frac{1-S_{1}^{*}(m)}{m}\right] P^{(2)}(0, z)+\alpha z\left[\frac{1-S_{2}^{*}(m)}{m}\right] P^{(2)}(0, z) \\
& +\alpha z\left[\frac{1-S_{3}^{*}(m)}{m}\right] P^{(3)}(0, z)-R(0, z)=-\alpha Q
\end{aligned}
$$

Let $\lambda-\lambda C(z)+\alpha=m$ and $\lambda-\lambda C(z)+\gamma-\gamma / z=k$. Putting Equations (51)-(53) into Equation (38) to obtain

$$
V(0, z)=\phi\left[P^{(1)}(0, z) S_{1}^{*}(m)+P^{(2)}(0, z) S_{2}^{*}(m)+P^{(3)}(0, z) S_{3}^{*}(m)\right]
$$

Substituting $V(0, z)$ of Equation (60) into Equations (56), (57) and (58) leads to

$$
\begin{aligned}
& {\left[z-p_{1} S_{1}^{*}(m)\left\{(1-\phi)+\phi F^{*}(k)\right\}\right] P^{(1)}(0, z)} \\
& -p_{2} S_{2}^{*}(m)\left\{(1-\phi)+\phi F^{*}(k)\right\} P^{(2)}(0, z) \\
& -p_{2} S_{3}^{*}(m)\left\{(1-\phi)+\phi F^{*}(k)\right\} P^{(3)}(0, z)-p_{1} B^{*}(k) R(0, z)=-p_{1} m Q
\end{aligned}
$$




$$
\begin{aligned}
& {\left[z-p_{2} S_{2}^{*}(m)\left\{(1-\phi)+\phi F^{*}(k)\right\}\right] P^{(2)}(0, z)} \\
& -p_{2} S_{1}^{*}(m)\left\{(1-\phi)+\phi F^{*}(k)\right\} P^{(1)}(0, z) \\
& -p_{2} S_{3}^{*}(m)\left\{(1-\phi)+\phi F^{*}(k)\right\} P^{(3)}(0, z)-p_{2} B^{*}(k) R(0, z)=-p_{2} m Q \\
& {\left[z-p_{3} S_{3}^{*}(m)\left\{(1-\phi)+\phi F^{*}(k)\right\}\right] P^{(3)}(0, z)} \\
& -p_{3} S_{1}^{*}(m)\left\{(1-\phi)+\phi F^{*}(k)\right\} P^{(1)}(0, z) \\
& -p_{3} S_{2}^{*}(m)\left\{(1-\phi)+\phi F^{*}(k)\right\} P^{(2)}(0, z)-p_{3} B^{*}(k) R(0, z)=-p_{3} m Q
\end{aligned}
$$

Solving Equations (59), (61), (62) and (63) simultaneously gives

$$
\begin{gathered}
P^{(1)}(0, z)=\frac{z p_{1}\left[m-\alpha B^{*}(k)\right] Q}{D(z)} \\
P^{(2)}(0, z)=\frac{z p_{2}\left[m-\alpha B^{*}(k)\right] Q}{D(z)} \\
P^{(3)}(0, z)=\frac{z p_{3}\left[m-\alpha B^{*}(k)\right] Q}{D(z)} \\
R(0, z)=\frac{z \alpha Q\left\{p_{1} S_{1}^{*}(m)+p_{2} S_{2}^{*}(m)+p_{3} S_{3}^{*}(m)\right\}\left\{\left[(1-\phi)+\phi F^{*}(k)\right]-z\right\}}{D(z)}
\end{gathered}
$$

Now, substituting Equations (64), (65) and (66) into Equation (60), one gets

$$
V(0, z)=\frac{z \phi Q\left[m-\alpha B^{*}(k)\right]\left[p_{1} S_{1}^{*}(m)+p_{2} S_{2}^{*}(m)+p_{3} S_{3}^{*}(m)\right]}{D(z)}
$$

where

$$
\begin{aligned}
D(z)= & z^{2}-z\left\{(1-\phi)+\phi F^{*}(k)\right\}\left\{p_{1} S_{1}^{*}(m)+p_{2} S_{2}^{*}(m)+p_{3} S_{3}^{*}(m)\right\} \\
& -z^{2} \alpha B^{*}(k)\left\{\frac{p_{1}\left[1-S_{1}^{*}(m)\right]}{m}+\frac{p_{2}\left[1-S_{2}^{*}(m)\right]}{m}+\frac{p_{3}\left[1-S_{3}^{*}(m)\right]}{m}\right\}
\end{aligned}
$$

Substituting Equation (64) into Equation (41); Equation (65) into Equation (42); Equation (66) into Equation (43), Equation (67) into Equation (44) and Equation (68) into Equation (45), the following are obtained:

$$
\begin{gathered}
P^{(1)}(z)=\frac{z p_{1}\left[m-\alpha B^{*}(k)\right] Q}{D(z)}\left[\frac{1-S_{1}^{*}(m)}{m}\right] \\
P^{(2)}(z)=\frac{z p_{2}\left[m-\alpha B^{*}(k)\right] Q}{D(z)}\left[\frac{1-S_{2}^{*}(m)}{m}\right] \\
P^{(3)}(z)=\frac{z p_{3}\left[m-\alpha B^{*}(k)\right] Q}{D(z)}\left[\frac{1-S_{3}^{*}(m)}{m}\right] \\
\left.\left.R(z)=\frac{z \alpha Q\left\{p_{1} S_{1}^{*}(m)+p_{2} S_{2}^{*}(m)+p_{3} S_{3}^{*}(m)\right\}\left[\left\{(1-\phi)+\phi F^{*}(k)\right\}-z\right]}{D(z)}\right] \frac{1-B^{*}(k)}{k}\right]
\end{gathered}
$$




$$
V(z)=\frac{z \phi Q\left[m-\alpha B^{*}(k)\right]\left[p_{1} S_{1}^{*}(m)+p_{2} S_{2}^{*}(m)+p_{3} S_{3}^{*}(m)\right]}{D(z)}\left[\frac{1-F^{*}(k)}{k}\right]
$$

where $D(z)$ has been defined in Equation (69).

Corollary 1. For the proposed queueing model, the steady-state probabilities that the server is active providing service to customers in two fluctuating modes, 1, 2 and 3 at any random point of time are given by Equations (75)-(77).

Proof. Since $P_{1}(z), P_{2}(z)$ and $P_{3}(z)$ are of indeterminate of the $0 / 0$ form when $z=1$, by applying L'Hopital's rule on Equations (70), (71) and (72) respectively, gives

$$
\begin{aligned}
P^{(1)}(1)= & \lim _{z \rightarrow 1} P^{(1)}(z) \\
= & \left(Q p_{1}[\lambda E(I)+\alpha(\lambda E(I)-\gamma) E(R)]\left[\frac{1-S_{1}^{*}(m)}{m}\right]\right) \\
& \times\left(\left[p_{1} S_{1}^{*}(\alpha)+p_{2} S_{2}^{*}(\alpha)+p_{3} S_{3}^{*}(\alpha)\right]\{1-\phi(\lambda E(I)-\gamma) E(V)\}\right. \\
& -[\alpha(\lambda E(I)-\gamma) E(R)+\lambda E(I)]\left\{\frac{p_{1}\left(1-S_{1}^{*}(\alpha)\right)}{\alpha}\right. \\
& \left.\left.+\frac{p_{2}\left(1-S_{2}^{*}(\alpha)\right)}{\alpha}+\frac{p_{3}\left(1-S_{3}^{*}(\alpha)\right)}{\alpha}\right\}\right)^{-1} \\
P^{(2)}(1)= & \lim _{z \rightarrow 1} P^{(2)}(z) \\
= & \left(Q p_{2}[\lambda E(I)+\alpha(\lambda E(I)-\gamma) E(R)]\left[\frac{1-S_{2}^{*}(m)}{m}\right]\right) \\
& \times\left(\left[p_{1} S_{1}^{*}(\alpha)+p_{2} S_{2}^{*}(\alpha)+p_{3} S_{3}^{*}(\alpha)\right]\{1-\phi(\lambda E(I)-\gamma) E(V)\}\right. \\
& \left.\left.\left.+\frac{p_{2}\left(1-S_{2}^{*}(\alpha)\right)}{\alpha}+\frac{p_{3}\left(1-S_{3}^{*}(\alpha)\right)}{\alpha}\right\}\right)^{*}(\alpha)+p_{2} S_{2}^{*}(\alpha)+p_{3} S_{3}^{*}(\alpha)\right]\{1-\phi(\lambda E(I)-\gamma) E(V)\} \\
& -[\alpha(\lambda E(I)-\gamma) E(R)+\lambda E(I)]\left\{\frac{p_{1}\left(1-S_{1}^{*}(\alpha)\right)}{\alpha}\right. \\
P^{(3)}(1)= & \lim _{z \rightarrow 1} P^{(3)}(z) \\
= & \left.Q p_{3}[\lambda E(I)+\alpha(\lambda E(I)-\gamma) E(R)]\left[\frac{1-S_{3}^{*}(m)}{m}\right]\right) \\
& (\alpha)
\end{aligned}
$$




$$
\begin{aligned}
& -[\alpha(\lambda E(I)-\gamma) E(R)+\lambda E(I)]\left\{\frac{p_{1}\left(1-S_{1}^{*}(\alpha)\right)}{\alpha}\right. \\
& \left.\left.+\frac{p_{2}\left(1-S_{2}^{*}(\alpha)\right)}{\alpha}+\frac{p_{3}\left(1-S_{3}^{*}(\alpha)\right)}{\alpha}\right\}\right)^{-1}
\end{aligned}
$$

where $E(I)$ is the mean size of batch of arriving customers, $E(R)$ is the mean repair time $E(V)$ is the mean of vacation time,

$G_{1}^{*}(0)=G_{2}^{*}(0)=G_{3}^{*}(0)=1$ and $V^{*}(0)=1$.

Corollary 2. For the proposed queueing model, the steady-state probability that the server is in failed state and is under repairs is given by Equation (78).

Proof. Since $R(z)$ is of indeterminate of the $0 / 0$ form when $z=1$, by applying L'Hopital's rule on Equation (73), one gets

$$
\begin{aligned}
R(1)= & \lim _{z \rightarrow 1} R(z) \\
= & \left(\alpha Q\left[p_{1} S_{1}^{*}(\alpha)+p_{2} S_{2}^{*}(\alpha)+p_{3} S_{3}^{*}(\alpha)\right][1-(\lambda E(I)-\gamma) \phi E(V)] E(R)\right) \\
& \times\left(\left[p_{1} S_{1}^{*}(\alpha)+p_{2} S_{2}^{*}(\alpha)+p_{3} S_{3}^{*}(\alpha)\right]\{1-\phi(\lambda E(I)-\gamma) E(V)\}\right. \\
& -[\alpha(\lambda E(I)-\gamma) E(R)+\lambda E(I)]\left\{\frac{p_{1}\left(1-S_{1}^{*}(\alpha)\right)}{\alpha}\right. \\
& \left.\left.+\frac{p_{2}\left(1-S_{2}^{*}(\alpha)\right)}{\alpha}+\frac{p_{3}\left(1-S_{3}^{*}(\alpha)\right)}{\alpha}\right\}\right)^{-1}
\end{aligned}
$$

Corollary 3. For the proposed queueing model, the steady-state probability that the server goes on vacation at any random point of time is given by Equation (79).

Proof. Since $V(z)$ is of indeterminate of the $0 / 0$ form when $z=1$, by applying L'Hopital's rule on Equation (74), the following is obtained

$$
\begin{aligned}
V(1)= & \lim _{z \rightarrow 1} V(z) \\
= & \left(Q \phi[\lambda E(I)+\alpha(\lambda E(I)-\gamma) E(R)]\left[p_{1} S_{1}^{*}(\alpha)+p_{2} S_{2}^{*}(\alpha)+p_{3} S_{3}^{*}(\alpha)\right] E(V)\right) \\
& \times\left(\left[p_{1} S_{1}^{*}(\alpha)+p_{2} S_{2}^{*}(\alpha)+p_{3} S_{3}^{*}(\alpha)\right]\{1-\phi(\lambda E(I)-\gamma) E(V)\}\right. \\
& -[\alpha(\lambda E(I)-\gamma) E(R)+\lambda E(I)]\left\{\frac{p_{1}\left(1-S_{1}^{*}(m)\right)}{\alpha}\right. \\
& \left.\left.+\frac{p_{2}\left(1-S_{2}^{*}(m)\right)}{\alpha}+\frac{p_{3}\left(1-S_{3}^{*}(m)\right)}{\alpha}\right\}\right)^{-1}
\end{aligned}
$$

Theorem 2. For the proposed queueing model, the probability generating function of the queue size irrespective of the state of the system, denoted by 
$P_{q}(z)$, is given by Equation (80).

Proof. Adding Equations (70), (71), (72), (73) and (74) leads to:

$$
\begin{aligned}
& P_{q}(z)=\frac{z\left[m-\alpha B^{*}(k)\right] Q}{D(z)}\left[\frac{p_{1}\left(1-S_{1}^{*}(m)\right)+p_{3}\left(1-S_{2}^{*}(m)\right)+p_{3}\left(1-S_{1}^{*}(m)\right)}{m}\right] \\
& \left.\left.+\frac{z \alpha Q\left\{p_{1} S_{1}^{*}(m)+p_{2} S_{2}^{*}(m)+p_{3} S_{3}^{*}(m)\right\}\left[\left\{(1-\phi)+\phi F^{*}(k)\right\}-z\right]}{D(z)}\right] \frac{1-B^{*}(k)}{k}\right] \\
& +\frac{z \phi Q\left[m-\alpha B^{*}(k)\right]\left[p_{1} S_{1}^{*}(m)+p_{2} S_{2}^{*}(m)+p_{3} S_{3}^{*}(m)\right]}{D(z)}\left[\frac{1-F^{*}(k)}{k}\right]
\end{aligned}
$$

where $D(z)$ is defined in Equation (69).

\subsection{Performance Measures of the Proposed Queueing Model}

Theorem 3. For the proposed queueing model, the probability that the server is idle, denoted by $Q$, is given by Equation (81).

Proof. Adding Equations (75), (76), (77), (78) and (79) and substituting the result into the normalizing condition $Q+P_{q}(1)=1$ yields

$$
Q=\frac{-[\alpha(\lambda E(I)-\gamma) E(R)+\lambda E(I)]\left\{\frac{p_{1}\left(1-S_{1}^{*}(\alpha)\right)}{\alpha}+\frac{p_{2}\left(1-S_{2}^{*}(\alpha)\right)}{\alpha}+\frac{p_{3}\left(1-S_{3}^{*}(\alpha)\right)}{\alpha}\right\}}{[1+\phi \gamma E(V)+\alpha E(R)]\left[\left\{p_{1} S_{1}^{*}(\alpha)+p_{2} S_{2}^{*}(\alpha)+p_{3} S_{3}^{*}(\alpha)\right\}\right]}
$$

Theorem 4. For the proposed queueing model, the probability that the server is busy (utilization factor), denoted by $\rho$, is given by Equation (82).

Proof. Substitution of Equation (81) into the relation $\rho=1-Q$ gives

$$
\begin{gathered}
{[\alpha(\lambda E(I)-\gamma) E(R)+\lambda E(I)]\left\{\frac{p_{1}\left(1-S_{1}^{*}(\alpha)\right)}{\alpha}+\frac{p_{2}\left(1-S_{2}^{*}(\alpha)\right)}{\alpha}+\frac{p_{3}\left(1-S_{3}^{*}(\alpha)\right)}{\alpha}\right\}} \\
\rho=\frac{+[\phi \lambda E(I) E(V)-\alpha E(R)]\left[p_{1} S_{1}^{*}(\alpha)+p_{2} S_{2}^{*}(\alpha)+p_{3} S_{3}^{*}(\alpha)\right]}{[1+\phi \gamma E(V)+\alpha E(R)]\left[\left\{p_{1} S_{1}^{*}(\alpha)+p_{2} S_{2}^{*}(\alpha)+p_{3} S_{3}^{*}(\alpha)\right\}\right]}
\end{gathered}
$$

\section{Special Cases of the Proposed Queueing Model}

Some of sub-models of the proposed queuing model are:

Case 1: Batch arrival single server vacation queue with server providing service in three fluctuating modes with breakdown

Suppose we let the reneging parameter $\gamma=0$ in the proposed queueing model, one gets the results in Case 2.

Case 2: Batch arrival single server vacation queue with server providing service in three fluctuating modes with reneging

By letting the breakdown parameter $\alpha=0$ in the proposed queueing model, we get the results of Case 2.

Case 3: Batch arrival single server vacation queue with server providing ser- 
vice in three fluctuating modes

By letting the breakdown parameter $\alpha=0$ and the reneging parameter $\gamma=0$ in the proposed queueing model, we obtain the model in Case 3 .

Case 4: Batch arrival single server queue with server providing service in three fluctuating modes with reneging during server breakdowns

Suppose we let the vacation parameter $\phi=0$ in the proposed queueing model, one obtains the model in Case 4.

Case 5: Batch arrival single server vacation queue with server providing service in two fluctuating modes during reneging during breakdowns

Case 5 is be obtained by letting the probability of server rendering service in the third mode $p_{3}=0$ in the proposed queueing model, we have Case 5.

Case 6: Batch arrival single server queue with server providing service in two fluctuating modes (no breakdown, no vacation and no reneging). The corresponding results for this particular case are obtained by putting breakdown parameter $\alpha=0$, vacation parameter $\phi=0$ and reneging parameter $\gamma=0$.

Case 7: Batch arrival single server queueing model with two fluctuating models of service

We obtain Case 7 by putting $\alpha=0, \phi=0, \gamma=0, p_{3}=0$ in the proposed queueing model.

Case 8: Exponential service times, vacation time and repair time

The exponential distribution usually serves as the distribution of the service times, vacation time and repair time in batch arrival queueing modelling. Consequently, we define

$$
\left.\begin{array}{l}
S_{1}^{*}(m)=\mu_{1} /\left(\mu_{1}+m\right) \\
S_{2}^{*}(m)=\mu_{2} /\left(\mu_{2}+m\right) \\
S_{3}^{*}(m)=\mu_{3} /\left(\mu_{3}+m\right) \\
B^{*}(k)=\beta /(\beta+m) \\
F^{*}(k)=\eta /(\eta+m)
\end{array}\right\}
$$

where $m=\lambda-\lambda C(z)+\alpha$ and $k=\lambda-\lambda C(z)+\gamma-\gamma / z$. In addition, it is assumed that the units of arrivals are come one by one, such that $E(R)=1 / \beta$ and $E(V)=1 / \eta$. Thus, the result of the proposed queueing model reduces to:

1 ) probability that the server is providing service in mode 1 , mode 2 and mode 3 at a random point of time is are

$$
\begin{gathered}
P^{(1)}(1)=\frac{p_{1} Q\left[\lambda+\frac{\alpha(\lambda-\gamma)}{\beta}\right] \frac{1}{\mu_{1}+\alpha}}{\left[\frac{p_{1} \mu_{1}}{\mu_{1}+\alpha}+\frac{p_{2} \mu_{2}}{\mu_{2}+\alpha}+\frac{p_{3} \mu_{3}}{\mu_{3}+\alpha}\right]\left[1-\frac{\phi(\lambda-\gamma)}{\eta}\right]-\left[\lambda+\frac{\alpha(\lambda-\gamma)}{\beta}\right]\left(\frac{p_{1}}{\mu_{1}+\alpha}+\frac{p_{2}}{\mu_{2}+\alpha}+\frac{p_{3}}{\mu_{3}+\alpha}\right)} \\
P^{(2)}(1)=\frac{p_{2} Q\left[\lambda+\frac{\alpha(\lambda-\gamma)}{\beta}\right] \frac{1}{\mu_{2}+\alpha}}{\left[\frac{p_{1} \mu_{1}}{\mu_{1}+\alpha}+\frac{p_{2} \mu_{2}}{\mu_{2}+\alpha}+\frac{p_{3} \mu_{3}}{\mu_{3}+\alpha}\right]\left[1-\frac{\phi(\lambda-\gamma)}{\eta}\right]-\left[\lambda+\frac{\alpha(\lambda-\gamma)}{\beta}\right]\left(\frac{p_{1}}{\mu_{1}+\alpha}+\frac{p_{2}}{\mu_{2}+\alpha}+\frac{p_{3}}{\mu_{3}+\alpha}\right)}
\end{gathered}
$$




$$
P^{(3)}(1)=\frac{p_{3} Q\left[\lambda+\frac{\alpha(\lambda-\gamma)}{\beta}\right] \frac{1}{\mu_{3}+\alpha}}{\left[\frac{p_{1} \mu_{1}}{\mu_{1}+\alpha}+\frac{p_{2} \mu_{2}}{\mu_{2}+\alpha}+\frac{p_{3} \mu_{3}}{\mu_{3}+\alpha}\right]\left[1-\frac{\phi(\lambda-\gamma)}{\eta}\right]-\left[\lambda+\frac{\alpha(\lambda-\gamma)}{\beta}\right]\left(\frac{p_{1}}{\mu_{1}+\alpha}+\frac{p_{2}}{\mu_{2}+\alpha}+\frac{p_{3}}{\mu_{3}+\alpha}\right)}
$$

2) probability that the server is under repairs at random point of time is

$$
R(1)=\frac{\alpha Q\left[\frac{p_{1} \mu_{1}}{\mu_{1}+m}+\frac{p_{2} \mu_{2}}{\mu_{2}+m}+\frac{p_{3} \mu_{3}}{\mu_{3}+m}\right]\left[1-\frac{\phi(\lambda-\gamma)}{\eta}\right] \frac{1}{\beta}}{\left[\frac{p_{1} \mu_{1}}{\mu_{1}+\alpha}+\frac{p_{2} \mu_{2}}{\mu_{2}+\alpha}+\frac{p_{3} \mu_{3}}{\mu_{3}+\alpha}\right]\left[1-\frac{\phi(\lambda-\gamma)}{\eta}\right]-\left[\lambda+\frac{\alpha(\lambda-\gamma)}{\beta}\right]\left(\frac{p_{1}}{\mu_{1}+\alpha}+\frac{p_{2}}{\mu_{2}+\alpha}+\frac{p_{3}}{\mu_{3}+\alpha}\right)}
$$

3) probability that the server is on vacation at random point of time is given by

$$
V(1)=\frac{\phi Q\left[\frac{p_{1} \mu_{1}}{\mu_{1}+\alpha}+\frac{p_{2} \mu_{2}}{\mu_{2}+\alpha}+\frac{p_{3} \mu_{3}}{\mu_{3}+\alpha}\right]\left[\lambda+\frac{\alpha(\lambda-\gamma)}{\beta}\right] \frac{1}{\eta}}{\left[\frac{p_{1} \mu_{1}}{\mu_{1}+\alpha}+\frac{p_{2} \mu_{2}}{\mu_{2}+\alpha}+\frac{p_{3} \mu_{3}}{\mu_{3}+\alpha}\right]\left[1-\frac{\phi(\lambda-\gamma)}{\eta}\right]-\left[\lambda+\frac{\alpha(\lambda-\gamma)}{\beta}\right]\left(\frac{p_{1}}{\mu_{1}+\alpha}+\frac{p_{2}}{\mu_{2}+\alpha}+\frac{p_{3}}{\mu_{3}+\alpha}\right)}
$$

4) the probability that the server is idle but available in the system is given by

$$
Q=\frac{\left(\frac{p_{1} \mu_{1}}{\mu_{1}+\alpha}+\frac{p_{2} \mu_{2}}{\mu_{2}+\alpha}+\frac{p_{3} \mu_{3}}{\mu_{3}+\alpha}\right)\left[1-\frac{\phi(\lambda-\gamma)}{\eta}\right]-\left[\lambda+\frac{\alpha(\lambda-\gamma)}{\beta}\right]\left(\frac{p_{1}}{\mu_{1}+\alpha}+\frac{p_{2}}{\mu_{2}+\alpha}+\frac{p_{3}}{\mu_{3}+\alpha}\right)}{\left(1+\frac{\phi \gamma}{\beta}+\frac{\alpha}{\beta}\right)\left(\frac{p_{1} \mu_{1}}{\mu_{1}+\alpha}+\frac{p_{2} \mu_{2}}{\mu_{2}+\alpha}+\frac{p_{3} \mu_{3}}{\mu_{3}+\alpha}\right)}
$$

5) the utilization factor is

$$
\rho=\frac{\left(\lambda+\frac{\alpha(\lambda-\gamma)}{\beta}\right)\left(\frac{p_{1}}{\mu_{1}+\alpha}+\frac{p_{2}}{\mu_{2}+\alpha}+\frac{p_{3}}{\mu_{3}+\alpha}\right)+\left(\frac{\phi \lambda}{\eta}-\frac{\alpha}{\beta}\right)\left(\frac{p_{1} \mu_{1}}{\mu_{1}+\alpha}+\frac{p_{2} \mu_{2}}{\mu_{2}+\alpha}+\frac{p_{3} \mu_{3}}{\mu_{3}+\alpha}\right)}{\left(1+\frac{\phi \gamma}{\beta}+\frac{\alpha}{\beta}\right)\left(\frac{p_{1} \mu_{1}}{\mu_{1}+\alpha}+\frac{p_{2} \mu_{2}}{\mu_{2}+\alpha}+\frac{p_{3} \mu_{3}}{\mu_{3}+\alpha}\right)}
$$

\section{Numerical Examples}

To demonstrate the effect of server vacation and reneging during breakdown and repair periods on the behaviour of a batch arrival queueing system providing service in three fluctuating modes, we consider the eight (8) special case of the proposed queueing model where all service times, vacation time, reneging and repair times are exponentially distributed. Further, we assume that the customers are arriving one by one, so that $E(I)=1$ and $E(I(I-1))=0$. We choose arbitrary values for the parameters such that the steady state condition $\rho \leq 1$ is satisfied. We consider the value 4 for $\lambda$, the values $8,10,12$ for $\mu_{1}$, $\mu_{2}$ and $\mu_{3}$, the values $2,3,4, \ldots, 8,9$ for the parameter $\alpha$, the values $6,8,11$, 14 for the parameter $\beta$, the values 2, 3, 4, 5 for parameters $\gamma$ and $\eta$, the values $0.34,0.33,0.33$ for the parameters $p_{1}, p_{2}$ and $p_{3}$, and 0.5 for the parameter $\phi$. Using the above parameter values in Equations (89), (90), (84), (85), (86), (87) and (88) gives the estimates of $Q, \rho, P^{(1)}(1), P^{(2)}(1), P^{(3)}(1)$, $R(1)$ and $V(1)$ respectively, which are provided in Tables 1-4. From Tables $1-4$, it appears that when the rate of rate of system breakdown and repair are 
Table 1. Computed values of various steady-state characteristics of the proposed queueing model when $\beta=6$.

\begin{tabular}{|c|c|c|c|c|c|c|c|c|c|}
\hline$\alpha$ & $\gamma$ & $\eta$ & $Q$ & $\rho$ & $P^{(1)}(1)$ & $P^{(2)}(1)$ & $P^{(3)}(1)$ & $R(1)$ & $V(1)$ \\
\hline & 2 & 5 & 0.2144 & 0.7856 & 0.1275 & 0.1031 & 0.0884 & 0.1778 & 0.3111 \\
\hline \multirow[t]{4}{*}{2} & 3 & 4 & 0.2721 & 0.7279 & 0.1121 & 0.0907 & 0.0777 & 0.1842 & 0.3421 \\
\hline & 4 & 3 & 0.3540 & 0.6460 & 0.0983 & 0.0795 & 0.0682 & 0.2000 & 0.4000 \\
\hline & 5 & 2 & 0.4995 & 0.5005 & 0.0858 & 0.0694 & 0.0595 & 0.2381 & 0.5238 \\
\hline & 2 & 5 & 0.1730 & 0.8270 & 0.1212 & 0.0995 & 0.0863 & 0.2400 & 0.3000 \\
\hline \multirow[t]{4}{*}{3} & 3 & 4 & 0.2369 & 0.7631 & 0.1039 & 0.0853 & 0.0739 & 0.2500 & 0.3214 \\
\hline & 4 & 3 & 0.3222 & 0.6778 & 0.0881 & 0.0724 & 0.0627 & 0.2727 & 0.3636 \\
\hline & 5 & 2 & 0.4653 & 0.5347 & 0.0738 & 0.0606 & 0.0525 & 0.3261 & 0.4565 \\
\hline & 2 & 5 & 0.1391 & 0.8609 & 0.1161 & 0.0966 & 0.0845 & 0.2909 & 0.2909 \\
\hline \multirow[t]{4}{*}{4} & 3 & 4 & 0.2078 & 0.7922 & 0.0972 & 0.0808 & 0.0707 & 0.3043 & 0.3043 \\
\hline & 4 & 3 & 0.2957 & 0.7043 & 0.0798 & 0.0664 & 0.0581 & 0.3333 & 0.3333 \\
\hline & 5 & 2 & 0.4365 & 0.5635 & 0.0639 & 0.0531 & 0.0465 & 0.4000 & 0.4000 \\
\hline & 2 & 5 & 0.1109 & 0.8891 & 0.1119 & 0.0941 & 0.0831 & 0.3333 & 0.2833 \\
\hline \multirow[t]{4}{*}{5} & 3 & 4 & 0.1833 & 0.8167 & 0.0916 & 0.0771 & 0.0680 & 0.3500 & 0.2900 \\
\hline & 4 & 3 & 0.2732 & 0.7268 & 0.0729 & 0.0613 & 0.0541 & 0.3846 & 0.3077 \\
\hline & 5 & 2 & 0.3749 & 0.6251 & 0.0556 & 0.0468 & 0.0413 & 0.4321 & 0.2346 \\
\hline & 2 & 5 & 0.0870 & 0.9130 & 0.1084 & 0.0920 & 0.0818 & 0.3692 & 0.2769 \\
\hline \multirow[t]{4}{*}{6} & 3 & 4 & 0.1624 & 0.8376 & 0.0870 & 0.0739 & 0.0657 & 0.3889 & 0.2778 \\
\hline & 4 & 3 & 0.2539 & 0.7461 & 0.0671 & 0.0570 & 0.0506 & 0.4286 & 0.2857 \\
\hline & 5 & 2 & 0.3907 & 0.6093 & 0.0486 & 0.0413 & 0.0367 & 0.5172 & 0.3103 \\
\hline & 2 & 5 & 0.0665 & 0.9335 & 0.1054 & 0.0902 & 0.0807 & 0.4000 & 0.2714 \\
\hline \multirow[t]{4}{*}{7} & 3 & 4 & 0.1444 & 0.8556 & 0.0830 & 0.0711 & 0.0636 & 0.4224 & 0.2672 \\
\hline & 4 & 3 & 0.2371 & 0.7629 & 0.0621 & 0.0532 & 0.0476 & 0.4667 & 0.2667 \\
\hline & 5 & 2 & 0.3722 & 0.6278 & 0.0426 & 0.0365 & 0.0326 & 0.5645 & 0.2742 \\
\hline & 2 & 5 & 0.0487 & 0.9513 & 0.1028 & 0.0887 & 0.0798 & 0.4267 & 0.2667 \\
\hline \multirow[t]{4}{*}{8} & 3 & 4 & 0.1287 & 0.8713 & 0.0796 & 0.0687 & 0.0618 & 0.4516 & 0.2581 \\
\hline & 4 & 3 & 0.2224 & 0.7776 & 0.0578 & 0.0499 & 0.0449 & 0.5000 & 0.2500 \\
\hline & 5 & 2 & 0.3559 & 0.6441 & 0.0374 & 0.0322 & 0.0290 & 0.6061 & 0.2424 \\
\hline & 2 & 5 & 0.0332 & 0.9668 & 0.1005 & 0.0873 & 0.0790 & 0.4500 & 0.2625 \\
\hline \multirow[t]{3}{*}{9} & 3 & 4 & 0.1149 & 0.8851 & 0.0766 & 0.0665 & 0.0602 & 0.4773 & 0.2500 \\
\hline & 4 & 3 & 0.2095 & 0.7906 & 0.0541 & 0.0470 & 0.0425 & 0.5294 & 0.2353 \\
\hline & 5 & 2 & 0.3415 & 0.6585 & 0.0328 & 0.0285 & 0.0258 & 0.6429 & 0.2143 \\
\hline
\end{tabular}


Table 2. Computed values of various steady state characteristics of the proposed queueing model when $\beta=8$.

\begin{tabular}{|c|c|c|c|c|c|c|c|c|c|}
\hline$\alpha$ & $\gamma$ & $\eta$ & $Q$ & $\rho$ & $P^{(1)}(1)$ & $P^{(2)}(1)$ & $P^{(3)}(1)$ & $R(1)$ & $V(1)$ \\
\hline & 2 & 5 & 0.2463 & 0.7537 & 0.1341 & 0.1085 & 0.0930 & 0.4267 & 0.2667 \\
\hline \multirow[t]{4}{*}{2} & 3 & 4 & 0.3056 & 0.6944 & 0.1211 & 0.0980 & 0.0840 & 0.4516 & 0.2581 \\
\hline & 4 & 3 & 0.3933 & 0.6067 & 0.1093 & 0.0884 & 0.0757 & 0.5000 & 0.2500 \\
\hline & 5 & 2 & 0.5540 & 0.4460 & 0.0983 & 0.0795 & 0.0682 & 0.6061 & 0.2424 \\
\hline & 2 & 5 & 0.2093 & 0.7907 & 0.1279 & 0.1051 & 0.0911 & 0.4500 & 0.2625 \\
\hline \multirow[t]{4}{*}{3} & 3 & 4 & 0.2735 & 0.7265 & 0.1131 & 0.0929 & 0.0805 & 0.4773 & 0.2500 \\
\hline & 4 & 3 & 0.3635 & 0.6365 & 0.0994 & 0.0817 & 0.0708 & 0.5294 & 0.2353 \\
\hline & 5 & 2 & 0.5209 & 0.4791 & 0.0868 & 0.0713 & 0.0618 & 0.6429 & 0.2143 \\
\hline & 2 & 5 & 0.1779 & 0.8221 & 0.1228 & 0.1022 & 0.0894 & 0.1455 & 0.3273 \\
\hline \multirow[t]{4}{*}{4} & 3 & 4 & 0.2461 & 0.7539 & 0.1064 & 0.0885 & 0.0775 & 0.1522 & 0.3696 \\
\hline & 4 & 3 & 0.3379 & 0.6621 & 0.0912 & 0.0759 & 0.0664 & 0.1667 & 0.4444 \\
\hline & 5 & 2 & 0.4924 & 0.5076 & 0.0771 & 0.0641 & 0.0561 & 0.2000 & 0.6000 \\
\hline & 2 & 5 & 0.1510 & 0.849 & 0.1185 & 0.0997 & 0.0879 & 0.2000 & 0.3167 \\
\hline \multirow[t]{4}{*}{5} & 3 & 4 & 0.2224 & 0.7776 & 0.1008 & 0.0848 & 0.0748 & 0.2100 & 0.3500 \\
\hline & 4 & 3 & 0.3157 & 0.6843 & 0.0843 & 0.0709 & 0.0625 & 0.2308 & 0.4103 \\
\hline & 5 & 2 & 0.4674 & 0.5326 & 0.0688 & 0.0579 & 0.0511 & 0.2778 & 0.5370 \\
\hline & 2 & 5 & 0.1277 & 0.8723 & 0.1148 & 0.0975 & 0.0867 & 0.2462 & 0.3077 \\
\hline \multirow[t]{4}{*}{6} & 3 & 4 & 0.2016 & 0.7983 & 0.0959 & 0.0815 & 0.0724 & 0.2593 & 0.3333 \\
\hline & 4 & 3 & 0.2962 & 0.7038 & 0.0783 & 0.0665 & 0.0591 & 0.2857 & 0.3810 \\
\hline & 5 & 2 & 0.4455 & 0.5545 & 0.0617 & 0.0524 & 0.0466 & 0.3448 & 0.4828 \\
\hline & 2 & 5 & 0.1073 & 0.8927 & 0.1116 & 0.0956 & 0.0855 & 0.2857 & 0.3000 \\
\hline \multirow[t]{4}{*}{7} & 3 & 4 & 0.1836 & 0.8164 & 0.0918 & 0.0786 & 0.0703 & 0.3017 & 0.3190 \\
\hline & 4 & 3 & 0.2789 & 0.7211 & 0.0731 & 0.0626 & 0.0560 & 0.3333 & 0.3556 \\
\hline & 5 & 2 & 0.4260 & 0.5740 & 0.0555 & 0.0475 & 0.0425 & 0.4032 & 0.4355 \\
\hline & 2 & 5 & 0.0893 & 0.9107 & 0.1088 & 0.0939 & 0.0845 & 0.3200 & 0.2933 \\
\hline \multirow[t]{4}{*}{8} & 3 & 4 & 0.1675 & 0.8325 & 0.0881 & 0.0760 & 0.0684 & 0.3387 & 0.3065 \\
\hline & 4 & 3 & 0.2636 & 0.7364 & 0.0685 & 0.0591 & 0.0532 & 0.3750 & 0.3333 \\
\hline & 5 & 2 & 0.4086 & 0.5914 & 0.0500 & 0.0431 & 0.0388 & 0.4545 & 0.3939 \\
\hline & 2 & 5 & 0.0732 & 0.9268 & 0.1064 & 0.0924 & 0.0836 & 0.3500 & 0.2875 \\
\hline \multirow[t]{3}{*}{9} & 3 & 4 & 0.1531 & 0.8469 & 0.0849 & 0.0737 & 0.0667 & 0.3712 & 0.2955 \\
\hline & 4 & 3 & 0.2499 & 0.7501 & 0.0645 & 0.0560 & 0.0507 & 0.4118 & 0.3137 \\
\hline & 5 & 2 & 0.3929 & 0.6071 & 0.0452 & 0.0392 & 0.0355 & 0.5000 & 0.3571 \\
\hline
\end{tabular}


Table 3. Computed values of various steady state characteristics of the proposed queueing model when $\beta=11$.

\begin{tabular}{|c|c|c|c|c|c|c|c|c|c|}
\hline$\alpha$ & $\gamma$ & $\eta$ & $Q$ & $\rho$ & $P^{(1)}(1)$ & $P^{(2)}(1)$ & $P^{(3)}(1)$ & $R(1)$ & $V(1)$ \\
\hline & 2 & 5 & 0.2771 & 0.7229 & 0.1405 & 0.1136 & 0.0974 & 0.1143 & 0.3429 \\
\hline \multirow[t]{4}{*}{2} & 3 & 4 & 0.3386 & 0.6614 & 0.1300 & 0.1051 & 0.0901 & 0.1207 & 0.3966 \\
\hline & 4 & 3 & 0.4326 & 0.5674 & 0.1202 & 0.0972 & 0.0833 & 0.1333 & 0.4889 \\
\hline & 5 & 2 & 0.6093 & 0.3907 & 0.1110 & 0.0898 & 0.0770 & 0.1613 & 0.6774 \\
\hline & 2 & 5 & 0.2456 & 0.7544 & 0.1347 & 0.1106 & 0.0958 & 0.1600 & 0.3333 \\
\hline \multirow[t]{4}{*}{3} & 3 & 4 & 0.3107 & 0.6893 & 0.1225 & 0.1006 & 0.0872 & 0.1694 & 0.3790 \\
\hline & 4 & 3 & 0.4061 & 0.5939 & 0.1111 & 0.0912 & 0.0791 & 0.1875 & 0.4583 \\
\hline & 5 & 2 & 0.5791 & 0.4209 & 0.1004 & 0.0824 & 0.0714 & 0.2273 & 0.6212 \\
\hline & 2 & 5 & 0.2179 & 0.7821 & 0.1297 & 0.1079 & 0.0944 & 0.2000 & 0.3250 \\
\hline \multirow[t]{4}{*}{4} & 3 & 4 & 0.2861 & 0.7139 & 0.1161 & 0.0966 & 0.0845 & 0.2121 & 0.3636 \\
\hline & 4 & 3 & 0.3826 & 0.6174 & 0.1033 & 0.0859 & 0.0752 & 0.2353 & 0.4314 \\
\hline & 5 & 2 & 0.5522 & 0.4478 & 0.0912 & 0.0759 & 0.0664 & 0.2857 & 0.5714 \\
\hline & 2 & 5 & 0.1935 & 0.8065 & 0.1255 & 0.1055 & 0.0931 & 0.2353 & 0.3176 \\
\hline \multirow[t]{4}{*}{5} & 3 & 4 & 0.2643 & 0.7357 & 0.1106 & 0.0930 & 0.0821 & 0.2500 & 0.3500 \\
\hline & 4 & 3 & 0.3617 & 0.6383 & 0.0965 & 0.0812 & 0.0717 & 0.2778 & 0.4074 \\
\hline & 5 & 2 & 0.5281 & 0.4719 & 0.0833 & 0.0700 & 0.0618 & 0.3378 & 0.5270 \\
\hline & 2 & 5 & 0.1718 & 0.8282 & 0.1218 & 0.1034 & 0.0919 & 0.2667 & 0.3111 \\
\hline \multirow[t]{4}{*}{6} & 3 & 4 & 0.2448 & 0.7552 & 0.1058 & 0.0898 & 0.0798 & 0.2838 & 0.3378 \\
\hline & 4 & 3 & 0.3429 & 0.6571 & 0.0906 & 0.0770 & 0.0684 & 0.3158 & 0.3860 \\
\hline & 5 & 2 & 0.5065 & 0.4935 & 0.0763 & 0.0648 & 0.0576 & 0.3846 & 0.4872 \\
\hline & 2 & 5 & 0.1524 & 0.8476 & 0.1185 & 0.1015 & 0.0908 & 0.2947 & 0.3053 \\
\hline \multirow[t]{4}{*}{7} & 3 & 4 & 0.2273 & 0.7727 & 0.1015 & 0.0870 & 0.0778 & 0.3141 & 0.3269 \\
\hline & 4 & 3 & 0.3260 & 0.6740 & 0.0854 & 0.0731 & 0.0654 & 0.3500 & 0.3667 \\
\hline & 5 & 2 & 0.4870 & 0.5130 & 0.0701 & 0.0600 & 0.0537 & 0.4268 & 0.4512 \\
\hline & 2 & 5 & 0.1348 & 0.8652 & 0.1156 & 0.0998 & 0.0898 & 0.3200 & 0.3000 \\
\hline \multirow[t]{4}{*}{8} & 3 & 4 & 0.2115 & 0.7885 & 0.0978 & 0.0843 & 0.0759 & 0.3415 & 0.3171 \\
\hline & 4 & 3 & 0.3107 & 0.6893 & 0.0808 & 0.0697 & 0.0627 & 0.3810 & 0.3492 \\
\hline & 5 & 2 & 0.4692 & 0.5308 & 0.0645 & 0.0557 & 0.0501 & 0.4651 & 0.4186 \\
\hline & 2 & 5 & 0.1190 & 0.8810 & 0.1131 & 0.0982 & 0.0888 & 0.3429 & 0.2952 \\
\hline \multirow[t]{3}{*}{9} & 3 & 4 & 0.1971 & 0.8029 & 0.0944 & 0.0820 & 0.0742 & 0.3663 & 0.3081 \\
\hline & 4 & 3 & 0.2967 & 0.7033 & 0.0766 & 0.0665 & 0.0602 & 0.4091 & 0.3333 \\
\hline & 5 & 2 & 0.4530 & 0.5470 & 0.0596 & 0.0517 & 0.0468 & 0.5000 & 0.3889 \\
\hline
\end{tabular}


Table 4. Computed values of various steady state characteristics of the proposed queueing model when $\beta=14$.

\begin{tabular}{|c|c|c|c|c|c|c|c|c|c|}
\hline$\alpha$ & $\gamma$ & $\eta$ & $Q$ & $\rho$ & $P^{(1)}(1)$ & $P^{(2)}(1)$ & $P^{(3)}(1)$ & $R(1)$ & $V(1)$ \\
\hline & 2 & 5 & 0.2970 & 0.7030 & 0.1446 & 0.1170 & 0.1003 & 0.0941 & 0.3529 \\
\hline \multirow[t]{4}{*}{2} & 3 & 4 & 0.3602 & 0.6398 & 0.1358 & 0.1098 & 0.0941 & 0.1000 & 0.4143 \\
\hline & 4 & 3 & 0.4589 & 0.5412 & 0.1275 & 0.1031 & 0.0884 & 0.1111 & 0.5185 \\
\hline & 5 & 2 & 0.6467 & 0.3533 & 0.1196 & 0.0967 & 0.0829 & 0.1351 & 0.7297 \\
\hline & 2 & 5 & 0.2698 & 0.7302 & 0.1391 & 0.1143 & 0.0990 & 0.1333 & 0.3444 \\
\hline \multirow[t]{4}{*}{3} & 3 & 4 & 0.3358 & 0.6642 & 0.1288 & 0.1058 & 0.0917 & 0.1419 & 0.3986 \\
\hline & 4 & 3 & 0.4352 & 0.5648 & 0.1191 & 0.0978 & 0.0847 & 0.1579 & 0.4912 \\
\hline & 5 & 2 & 0.6193 & 0.3807 & 0.1098 & 0.0902 & 0.0782 & 0.1923 & 0.6795 \\
\hline & 2 & 5 & 0.2453 & 0.7547 & 0.1344 & 0.1118 & 0.0979 & 0.1684 & 0.3368 \\
\hline \multirow[t]{4}{*}{4} & 3 & 4 & 0.3138 & 0.6862 & 0.1228 & 0.1022 & 0.0894 & 0.1795 & 0.3846 \\
\hline & 4 & 3 & 0.4139 & 0.5861 & 0.1118 & 0.0930 & 0.0814 & 0.2000 & 0.4667 \\
\hline & 5 & 2 & 0.5945 & 0.4055 & 0.1012 & 0.0842 & 0.0737 & 0.2439 & 0.6341 \\
\hline & 2 & 5 & 0.2233 & 0.7767 & 0.1303 & 0.1096 & 0.0967 & 0.2000 & 0.3300 \\
\hline \multirow[t]{4}{*}{5} & 3 & 4 & 0.2939 & 0.7061 & 0.1175 & 0.0989 & 0.0872 & 0.2134 & 0.3720 \\
\hline & 4 & 3 & 0.3946 & 0.6054 & 0.1053 & 0.0886 & 0.0782 & 0.2381 & 0.4444 \\
\hline & 5 & 2 & 0.5719 & 0.4281 & 0.0937 & 0.0788 & 0.0695 & 0.2907 & 0.5930 \\
\hline & 2 & 5 & 0.2033 & 0.7967 & 0.1267 & 0.1076 & 0.0957 & 0.2286 & 0.3238 \\
\hline \multirow[t]{4}{*}{6} & 3 & 4 & 0.2759 & 0.7241 & 0.1129 & 0.0958 & 0.0852 & 0.2442 & 0.3605 \\
\hline & 4 & 3 & 0.3769 & 0.6231 & 0.0996 & 0.0846 & 0.0752 & 0.2727 & 0.4242 \\
\hline & 5 & 2 & 0.5513 & 0.4487 & 0.0870 & 0.0739 & 0.0657 & 0.3333 & 0.5556 \\
\hline & 2 & 5 & 0.1851 & 0.8149 & 0.1235 & 0.1058 & 0.0946 & 0.2545 & 0.3182 \\
\hline \multirow[t]{4}{*}{7} & 3 & 4 & 0.2594 & 0.7406 & 0.1087 & 0.0931 & 0.0833 & 0.2722 & 0.3500 \\
\hline & 4 & 3 & 0.3608 & 0.6392 & 0.0945 & 0.0809 & 0.0724 & 0.3043 & 0.4058 \\
\hline & 5 & 2 & 0.5324 & 0.4676 & 0.0809 & 0.0693 & 0.0620 & 0.3723 & 0.5213 \\
\hline & 2 & 5 & 0.1685 & 0.8315 & 0.1207 & 0.1041 & 0.0937 & 0.2783 & 0.3130 \\
\hline \multirow[t]{4}{*}{8} & 3 & 4 & 0.2442 & 0.7558 & 0.1050 & 0.0906 & 0.0815 & 0.2979 & 0.3404 \\
\hline & 4 & 3 & 0.3460 & 0.6540 & 0.0899 & 0.0776 & 0.0698 & 0.3333 & 0.3889 \\
\hline & 5 & 2 & 0.5150 & 0.4850 & 0.0755 & 0.0651 & 0.0586 & 0.4082 & 0.4898 \\
\hline & 2 & 5 & 0.1533 & 0.8467 & 0.1181 & 0.1025 & 0.0928 & 0.3000 & 0.3083 \\
\hline \multirow[t]{3}{*}{9} & 3 & 4 & 0.2303 & 0.7697 & 0.1016 & 0.0882 & 0.0798 & 0.3214 & 0.3316 \\
\hline & 4 & 3 & 0.3323 & 0.6677 & 0.0858 & 0.0745 & 0.0674 & 0.3600 & 0.3733 \\
\hline & 5 & 2 & 0.4989 & 0.5011 & 0.0706 & 0.0613 & 0.0555 & 0.4412 & 0.4608 \\
\hline
\end{tabular}


held constant with increasing rate of reneging and decreasing rate of vacation completion, then the utilization factor decreases and the idle time of the system increases. In addition, the three fluctuating modes of service experiences a decrease in it probabilities of rendering service. More so, the increase in server vacation results to an increase in the probability of server vacation. Technically, from our results, the probability that server is under repair reduces provided the repair parameter increases whenever there is high rate of breakdowns.

\section{Concluding Remarks}

In this article, we introduced and studied a batch arrival single server queueing model for providing service in three fluctuating modes during reneging, server breakdown and server vacation. The supplementary variable technique was adopted for the derivation of the probability generating functions of the states of the server under the steady state condition. We examined the effects of server vacation, reneging, breakdowns and repair on the utilization factor, the idle time, the probabilities of server providing service in three fluctuating modes, the probability that the system is under repair and the probability that the server is on vacation. Results presented in Tables 1-4 show that when the rate of system breakdown and rate of repair completion remain constant with an increasing rate of reneging and a decreasing rate of server vacation, the probability that the server is busy decreases while the probability that the server is idle increases. Additionally, the probabilities that the server is providing service in modes 1, 2 and 3 decreases due to an increase in reneging and a decrease in completion of server vacation. Also, the results show that the probabilities of the server being under repair and on vacation are on the decrease due to the effect of increasing rate of reneging and decreasing rate of vacation completion.

Generally, the results of this article stress that any organization whereby mode of service delivery is fluctuating should develop queueing systems that can make provisions for breakdowns and repair so as to improve the completion of server vacation and reduce the rate of reneging.

\section{Acknowledgements}

The authors are grateful to the editor and reviewers for their careful reading and valuable comments which greatly improved the quality of the article.

\section{Conflicts of Interest}

The authors declare no conflicts of interest regarding the publication of this paper.

\section{References}

[1] Baruah, M., Madan, K.C. and Eldabi, T. (2014) A Batch Arrival Single Server Queue with Server Providing General Service in Two Fluctuating Modes and Reneging during Vacation and Breakdowns. Journal of Probability and Statistics, 2014, Article ID: 319318. https://doi.org/10.1155/2014/319318 
[2] Madan, K.C. (2014) On a Single Server Queue with Arrivals in Batches of Variable Size, General Service in Three Fluctuating Modes, Balking, Random Breakdowns and Stand-By Server during Breakdown Periods. Revista Investigacion Opereracional, 35, 189-200.

[3] Yamuni, A., Sumitha, D. and Chandrika, K.U. (2016) Bulk Arrival Retrial Queue with Fluctuating Modes of Service, Immediate Feedback and Server Vacation. International Journal of Engineering Sciences and Research Technology, 3, 1-16.

[4] Yamuni, A., Kirupa, K. and Chandrika, K.U. (2016) Bulk Arrival Retrial Queue with Fluctuating Modes of Service, Immediate Feedback, Server Vacation and Orbital Search. Global Journal of Engineering Science and Researches, 53, 760-768.

[5] Sowmiya, S. and Chandrika, K.U. (2016) Batch Arrival Retrial Queue with Fluctuating Modes of Service, Randomized Vacations and Orbital Search. International Journal of Latest Trends in Engineering and Technology, 7, 777-785.

[6] Rajadurai, P., Venkatesh, S. and Parameswari, K. (2018) An M/G/1 Retrial Queue with Fluctuating Modes of Services. International Journal of Engineering and Technology, 7, 762-765. https://doi.org/10.14419/ijet.v7i4.10.26110

[7] Ayyappan, G. and Supraja, R. (2018) An $M^{x} / G(a, b) / 1$ Queueing System with Two Fluctuating Modes of Service under Benoulli Vacation Schedule for Unreliable Server and Delaying Repair. International Journal of Pure and Applied Mathematics, 120, 49-57.

[8] Li, W., Shi, D. and Chao, X. (1997) Reliability Analysis of M/G/1 Queueing Systems with Server Breakdowns and Vacations. Journal of Applied Probability, 34, 546-555. https://doi.org/10.2307/3215393

[9] Tang, Y. (2003) Single Server M/G/1 Queuing System Subject to Breakdowns-Some Reliability and Queuing Problems. Microelectronics Reliability, 149, 588-613.

[10] Gray, W.J., Wang, P.P. and Scott, M. (2004) A Queuing Model with Multiple Types of Server Breakdowns. Quality Technology and Quantitative Management, 1, 245-255. https://doi.org/10.1080/16843703.2004.11673076

[11] Wang, J. (2004) An M/G/1 Queue with Second Optional Service and Server Breakdown. An International Journal Computers and Mathematics with Applications, 47, 1713-1723. https://doi.org/10.1016/j.camwa.2004.06.024

[12] Khalaf, R.F., Madan, K.C. and Lukas, C.A. (2011) An $M^{[x]} / G / 1$ Queue with Bernoulli Schedule Vacation Times, Random Breakdowns, General Delay Times and General Repair Times. Applied Mathematical Sciences, 5, 35-51.

[13] Ibe, O.C. (2015) M/G/1 Vacation Queueing Systems with Server Timeout. American Journal of Operations Research, 5, 77-88.

https://doi.org/10.4236/ajor.2015.52007

[14] Levy, Y. and Yechiali, U. (1975) Utilization of Idle Time in M/G/1 Queueing System. Management Sciences, 22, 202-211. https://doi.org/10.1287/mnsc.22.2.202

[15] Doshi, B. (1986) Queueing Systems with Vacations: A Survey. Queueing Systems, 1, 29-66. https://doi.org/10.1007/BF01149327

[16] Doshi, B.T. (1991) Analysis of a Two Phase Queueing System with General Service Times. Operations Research Letters, 10, 265-275. https://doi.org/10.1016/0167-6377(91)90012-E

[17] Tagaki, H. (1991) Queueing Analysis: A Foundation of Performance Evaluation Vacation and Priority Systems. North Holland, Amsterdam.

[18] Servi, L.D. and Finn, S.G. (2002) M/M/1 Queues with Working Vacations (M/ 
M/1/WV). Performance Evaluation, 50, 41-52.

https://doi.org/10.1016/S0166-5316(02)00057-3

[19] Tian, N. and Zhang, G. (2006) Vacation Queueing Models: Theory and Application. Springer-Verlag, New York. https://doi.org/10.1007/978-0-387-33723-4

[20] Wu, D. and Tagaki, H. (2006) M/G/1 Queue with Multiple Working Vacation. Performance Evaluation, 63, 654-681. https://doi.org/10.1016/j.peva.2005.05.005

[21] Banik, A., Gupta, U. and Pathak, S. (2007) On the GI/M/1/N Queue with Multiple Working Vacations-Analytic Analysis and Computation. Applied Mathematical Modelling, 31, 1701-1710. https://doi.org/10.1016/j.apm.2006.05.010

[22] Barrerm, D.Y. (1957) Queuing with Impatient Customers and Ordered Service. Operations Research, 5, 650-656. https://doi.org/10.1287/opre.5.5.650

[23] Haight, F.A. (1959) Queueing with Reneging. Metrika, 2, 186-197. https://doi.org/10.1007/BF02613734

[24] Ancker Jr., C.J. and Gafarian, A.V. (1963) Some Queueing Problems with Balking and Reneging. Operations Research, 11, 88-100. https://doi.org/10.1287/opre.11.1.88

[25] Hagighi, A.M., Medhi, J. and Mohanty, S.G. (1986) On a Multiserver Markovian Queuing System with Balking and Reneging. Computer and Operations Research, 13, 421-425. https://doi.org/10.1016/0305-0548(86)90029-8

[26] Bae, J., Kim, S. and Lee E.Y. (2001) The Virtual Waiting Time of the M/G/1 Queue with Impatient Customers. Queueing Systems, 38, 485-494. https://doi.org/10.1023/A:1010956213680

[27] Zhang, Y., Yue D. and Yue, W. (2005) Analysis of M/M/1/N Queue with Balking, Reneging and Server Vacations. Proceedings of the 5th International Symposium OR and Its Applications, China Tebet, 9-13 August 2005, 37-47.

[28] Altman, E. and Yechiali, U.N. (2006) Analysis of Customers' Impatience in Queues with Server Vacations. Queueing Systems, 52, 261-279. https://doi.org/10.1007/s11134-006-6134-X

[29] Choudhury, A. and Medhi, P. (2011) Balking and Reneging in Multiserver Makovian Queuing Systems. International Journal of Mathematics in Operations Research, 3, 377-394. https://doi.org/10.1504/IJMOR.2011.040874 